\title{
Modeling of Ion Permeation in Calcium and Sodium Channel Selectivity Filters
}

\author{
P.S. Raj Ganesh, ${ }^{1}$ Baron Chanda, ${ }^{2}$ S.K. Gupta, ${ }^{1}$ M.K. Mathew, ${ }^{2 *}$ and Jayaraman Chandrasekhar ${ }^{3 *}$ \\ ${ }^{1}$ Department of Chemical Engineering, Indian Institute of Science, Bangalore, India \\ ${ }^{2}$ National Centre for Biological Sciences, GKVK campus, University of Agricultural Sciences, Bangalore, India \\ ${ }^{3}$ Department of Organic Chemistry, Indian Institute of Science, Bangalore, India
}

\begin{abstract}
Structure-function studies have shown that it is possible to convert a sodium channel to a calcium-selective channel by a single amino acid substitution in the selectivity filter locus. Ion permeation through the "model selectivity filter" was modeled with a reduced set of functional groups representative of the constituent amino acid side chains. Force-field minimizations were conducted to obtain the energy profile of the cations as they get desolvated and bind to the "model selectivity filter." The calculations suggest that the ion selectivity in the calcium channel is due to preferential binding, whereas in the sodium channel it is due to exclusion. Energetics of displacement of a bound cation from the calcium "model selectivity filter" by another cation suggest that "multi-ion mechanism" reduces the activation barrier for ion permeation. Thus, the simple model captures qualitatively most of the conduction characteristics of sodium and calcium channels. However, the computed barriers for permeation are fairly large, suggesting that ion interaction with additional residues along the transport path may be essential to effect desolvation. Proteins 2000;38:384-392. ๑ 2000 Wiley-Liss, Inc.
\end{abstract}

Key words: minimizations; single-ion; multi-ion; binding; energetics; solvation

\section{INTRODUCTION}

Voltage-gated ion channels are exquisitely selective for the ions they conduct-distinguishing among $\mathrm{Na}^{+}, \mathrm{K}^{+}$ and $\mathrm{Ca}^{2+}$, for instance. ${ }^{1}$ High specificity has traditionally been associated with high-affinity binding. However, the observation of flux rates of up to $10^{7}$ ions/second through individual channel proteins would appear to be incompatible with high-affinity binding and consequent slow release of the transported ion. ${ }^{2}$ One approach to resolving this paradox is to postulate two closely spaced binding sites with similar high affinities. When two cations are present, their mutual repulsion would reduce the effective binding affinity, leading to high flux rates. ${ }^{3,4}$ However, mutagenesis data on sodium and calcium channels point to a single ring of residues constituting a "selectivity filter." $" 6$

Structurally, the channels can be viewed as consisting of four subunits, each contributing one reentrant loop or $\mathrm{P}$ region to the conducting pore. Alterations of some residues within this loop have been shown to affect selectivity. The function of the residues constituting the "selectivity filter" are best understood in $\mathrm{Na}^{+}$and $\mathrm{Ca}^{2+}$ channels where a single residue at equivalent positions on each of the loops plays the most decisive role in selectivity. ${ }^{5,6}$

Although the chemical constituents of the selectivity filter are known, the exact molecular mechanism by which ion conduction occurs specifically is not understood. Theoretical studies are quite useful for assessing various hypotheses. For example, studies on gramicidin A have led to interesting insights. ${ }^{7-10}$ Typically, these studies of selectivity have relied on identifying the free energy profile of a single cation as it gets stripped of its coordinated water molecules and permeates through the pore. ${ }^{11}$ However, this would require specification of the structure of the pore at atomic resolution and simulations lasting several microseconds. In the absence of such structural detail for the calcium and sodium channels and the required computational power, we have tried to capture the key permeation characteristics by using a simplified computational model.

The "model selectivity filters" (MSF) for sodium and calcium ion channels have been represented by a minimal set of functional groups. The energetics of ion binding has been obtained by using empirical force field minimizations. In addition to the relative binding energies, we have also calculated detailed profiles along the permeation pathway to estimate the barriers associated with desolvation of the ion. These data have been used to distinguish between two modes of selectivity, i.e., preferential binding and exclusion. In the former, as seen in model calcium selectivity filter (MCSF), the MSF binds with both sodium and calcium ions, but more effectively with one of them. In the exclusion mode of selectivity (as seen with model sodium selectivity filter [MSSF]), binding occurs only with the selected ion. The computed results are of interest because the two mechanisms can be differentiated experimentally.

Next, we have evaluated the energy profiles for the transport of an ion across the MCSF in the presence of a second cation to obtain insights about the multi-ion mode of permeation. This mechanism is particularly important in channels that are selective because of preferential

\footnotetext{
*Correspondence to: M.K. Mathew, National Centre for Biological Sciences, GKVK campus, University of Agricultural Sciences, Bangalore 560065, India. E-mail: mathew@ncbs.res.in or J. Chandrasekhar, Department of Organic Chemistry, Indian Institute of Science, Bangalore 560065, India.
}

Received 22 April 1999; Accepted 12 October 1999 
binding. We have examined the extent to which the permeation barriers are altered in such systems because of simultaneous interaction of two cations with the MSF. We also critically consider the performance of the simple computational model employed. Additional factors that might play an important role in bringing down the activation barriers and thereby realistic flux rates are suggested.

\section{Choice of the Model and Computational Methodology}

Molecular mechanics calculations were conducted to obtain details of the potential energy profiles for ion permeation across model selectivity filters. The functional groups to represent the MSFs were chosen on the basis of the numerous mutagenesis experiments performed to determine the minimum requirements of calcium and sodium selectivity filters that give the same permeation characteristics of a wild-type channel..$^{5,6,12}$

In a sodium channel it has been shown that the presence of aspartate (D), glutamate (E), and lysine (K) in the selectivity filter region gives rise to sodium selectivity. The other amino acid residues are known to play only a minor role in selectivity. ${ }^{12}$ Hence, DEK are the essential determinants of selectivity for a sodium channel. On the other hand, four glutamates have been characterized as constituting the selectivity filter in wild-type calcium channels. But the presence of three glutamates in the selectivity filter region is enough to result in a channel with similar selectivity trends. ${ }^{13}$

The key chemical interactions due to aspartate and glutamate must arise from the carboxylate unit present in their side chains. ${ }^{12}$ Also, this assumption is supported by the fact that mutation of a glutamate by an aspartate in the calcium channel reduces the binding site affinity, but not the selectivity. ${ }^{13}$ Along the same lines, the alkyl ammonium group in the lysine side chain is expected to be important in determining its role in the selectivity filter. Therefore, we have chosen a combination of two acetate ions and a methylammonium ion to represent the chemical constituents of the model sodium selectivity filter (MSSF). Similarly, three acetate ions were chosen as the model calcium selectivity filter (MCSF).

The exact structures for the MSFs were obtained as follows. The cation was allowed to interact with the functional groups of the MSF, and the system was immersed in a spherical shell of water molecules with a radius of $10 \AA$. The solvation shell includes 138 water molecules. The energy was minimized with respect to all the atomic positions. This model corresponds to one extreme in which the backbone of the selectivity filter has been assumed to have sufficient conformational flexibility to optimally coordinate with any cation, native or otherwise. Such a structure is henceforth referred to as the flexible MSF

Realistically, the selectivity filter of a particular channel may have lesser flexibility. Studies on aqueous cationbinding proteins whose structures are known suggest that the binding site is optimized for the native cation and is sufficiently rigid to prevent optimal binding of other cations. ${ }^{14}$ For example, it has been recently suggested ${ }^{15}$ that the reason behind the selectivity in a $\mathrm{K}^{+}$-channel whose crystal structure has been solved is a rigid binding site, which is tuned only to bind potassium ions optimally. To model this possibility for the calcium channel, the following approach was adopted. The structure of the selectivity filter was decided by optimizing the geometry with a bound calcium ion. Binding of this unit to a sodium ion was studied by freezing the methyl groups and optimizing only the relative orientations of the carboxylate groups. This structure is referred to as the rigid MSF for the calcium channel, because it corresponds to the situation in which the restructuring of the backbone of the amino acid side chains to optimally bind to other rejected cations (sodium, in the present case) is not permitted. A similar strategy of using both flexible and rigid models was used earlier to understand the role of cation- $\pi$ interactions in the selectivity of a $\mathrm{K}^{+}$-channel. ${ }^{16}$

To obtain the energy profile for binding, a set of calculations was conducted in which the ion is pulled out of the MSF until it becomes exclusively coordinated with water. The pathway for ion permeation was chosen as the axis perpendicular to the plane formed by the three methyl carbon atoms in the selectivity filter. The reaction coordinate $\mathbf{r}$ corresponds to the distance of the ion in a given geometry relative to the position in which it optimally interacts with the MSF. For each value of $\mathbf{r}$, the positions of the ion and the methyl groups of the MSF are fixed, but all other geometrical parameters, including those of the solvating water molecules, were fully optimized. The resultant potential energy curve takes into account the change in the interaction of the ion with the MSF and the variations in coordination with water, in addition to reorganization of the MSF and solvent water. These data have been generated to examine the relative strengths of binding of an ion with different MSFs and also to estimate the magnitude of barriers involved in the binding process.

A second set of calculations was performed to model the energetics associated with the multi-ion mechanism of ion permeation in which a cation effectively dislodges another cation bound to the MCSF. ${ }^{3,4}$ For all the dual-ion calculations, the position of the methyl groups was fixed on the basis of geometry of the selectivity filter obtained when a calcium is optimally bound to MCSF. Potential energy profiles were computed for a range of structures with two ions placed on either side of the MSF along the axis perpendicular to the plane formed by the three methyl carbon atoms at varying distances. Each structure is characterized by two coordinates, i.e., the distance $\mathbf{r}_{\mathbf{1}}$ of the first cation relative to its position in the bound state, and distance $\mathbf{r}_{2}$ corresponding to the relative position of the second cation on the opposite side of the plane formed by the methyl carbon atoms of the MSF (shown in Fig. 1). For each structure, geometry optimization was conducted for all parameters, except the positions of the ions and the methyl groups. Thus, a two-dimensional grid of energies as a function of two coordinates $\mathbf{r}_{1}$ and $\mathbf{r}_{\mathbf{2}}$ was obtained. The resultant contour diagrams provide a basis for identifying the preferred mode of ion permeation in the presence of a 


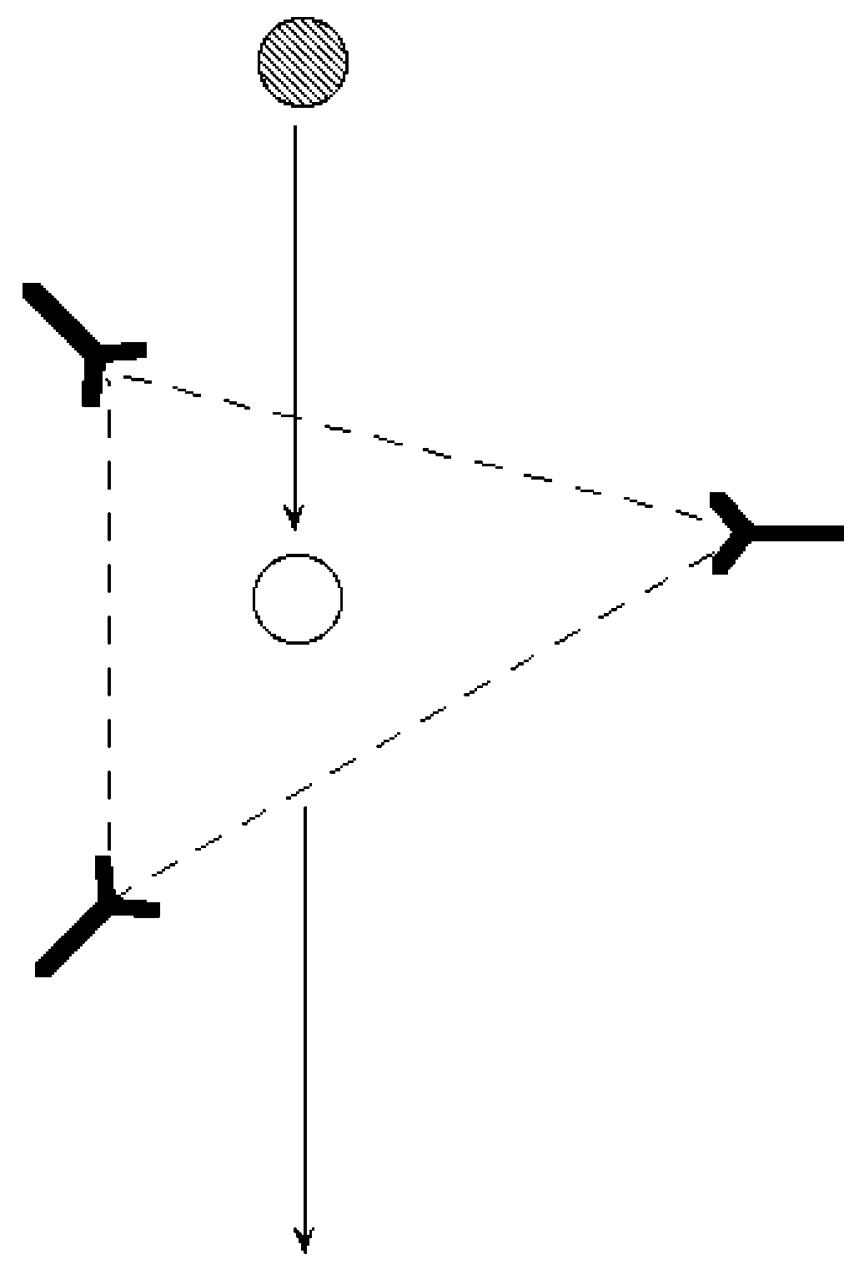

Fig. 1. A schematic representation of the model calcium selectivity filter in the presence of two $\mathrm{Ca}^{2+}$ ions. The plane formed by the methyl carbons of the three acetates is indicated with a dashed line. The ion permeation axis is perpendicular to the plane and is indicated with arrows. The figure shows the starting configuration with approaching ion at $r_{1}=5$ $\AA$ and the other ion in the filter at $r_{2}=0 \AA$. In the course of displacement, $r$. decreases from $5 \AA$ to $0 \AA$, whereas $r_{2}$ ranges from 0 to $-5 \AA$

second cation for a given MSF. The variations in activation barriers due to the presence of a second cation are also of interest.

All the energy minimizations were performed by using the DISCOVER module of BIOSYM (version 2.96). The $\mathrm{CVFF}^{17,18}$ was used for computing the energies. Minimizations were conducted by using the conjugate gradient method.

It is appropriate to summarize the key assumptions and simplifications used in the present work. The calculations correspond to a static model, designed to yield internal energy profiles. The interactions due to a large number of water molecules are included directly in the energy calculations. Although varying degrees of constraints are imposed on the constituents of the MSF, depending on the choice of rigid or flexible model, energy minimizations are conducted with respect to all solvent coordinates. The force field used here has been used widely for studying biomolecular structures ${ }^{19}$ and has been assessed with respect to accurate quantum chemical calculations. ${ }^{20}$ Although the precise energetics may vary with the choice of the force field, the key trends highlighted in this work are likely to be reliable. As is customary with many modern force fields in which the solvent molecules are included explicitly in the calculations, the dielectric constant of the medium is set to $1 .^{20}$ Traditionally, channel biophysicists have been influenced by the Born model, which places a high energetic cost for transferring an ion from water into a medium of low dielectric constant. ${ }^{21}$ This contribution is not taken into account in the present model, because the selectivity filter is chosen as a small polar unit. However, a more molecular level description of the energetic cost associated with desolvation of the ion during transport across the channel selectivity filter is included in the present work. The approach is consistent with the primary goal of the present study, i.e., to obtain insights into the origins of selectivity and relative flux, rather than absolute transport characteristics.

Although the tetrameric structure of the channel is well established, the mutagenesis data clearly indicate that selectivity is very tolerant of mutations in the residue on the fourth homology domain. ${ }^{12,13}$ Furthermore, experiments to understand molecular basis of proton block in calcium channels strongly suggest that glutamate in the fourth homology domain has a limited role in stabilization of the bound cation. ${ }^{22}$ Therefore, it is reasonable to assume that it may not participate in direct binding with the ion passing through. The use of three units for the selectivity filter rather than four is an approximation, which may only change the absolute energetics without affecting the selectivity trends. In our calculations, the binding site is optimized for the selected ion. This then results in the geometry that we use for our calculations. This is not necessarily inconsistent with the pseudotetrameric nature of the channels themselves as the elements of the selectivity filter need not be rigidly fixed to the center of each homology domain (or subunit) and so need not be at $90^{\circ}$ to each other.

\section{RESULTS AND DISCUSSION Energy Profiles for Single Ion Permeation}

Geometry optimization of the calcium ion bound to the MCSF and surrounded by 138 water molecules leads to a highly stabilized structure. The immediate coordination shell around the calcium ion (ligands that are within a $3 \AA$ sphere around the cation) is shown in Figure 2A. In addition to the six oxygen atoms of the ring of carboxylate ions, two water molecules are also found to be coordinated to the cation. The model selectivity filter obtained thus is defined as flexible MCSF. Rigid model selectivity filter is invoked only when considering the binding of a non-native cation to a selectivity filter (also see section on computational methodology).

The binding energy profile between the cation and the flexible MCSF was computed by restraining the ion at various distances $\mathbf{r}$ along an axis orthogonal to the ring of acetates. Two notable features are found in the resultant energy profile (Fig. 3). Initially, as $\mathbf{r}$ varies from $0 \AA$ (ion 
A

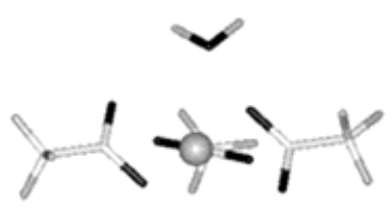

C

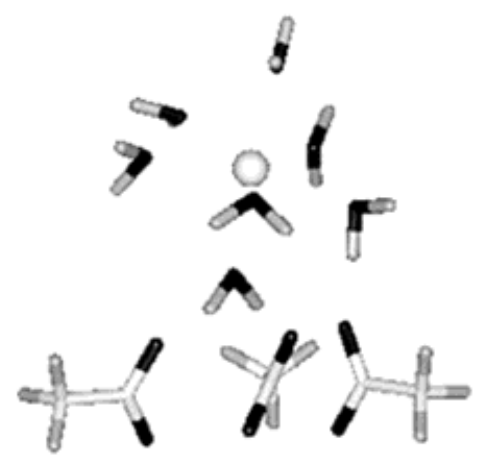

D

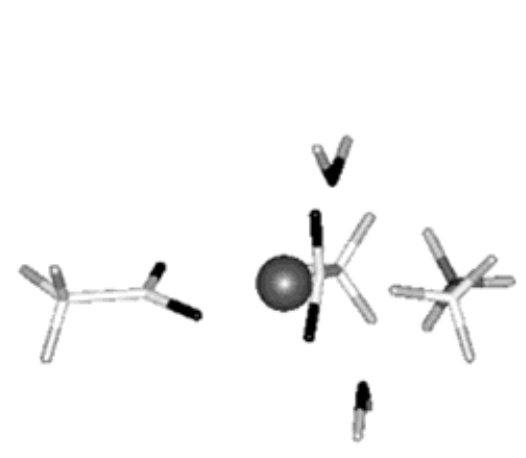

B
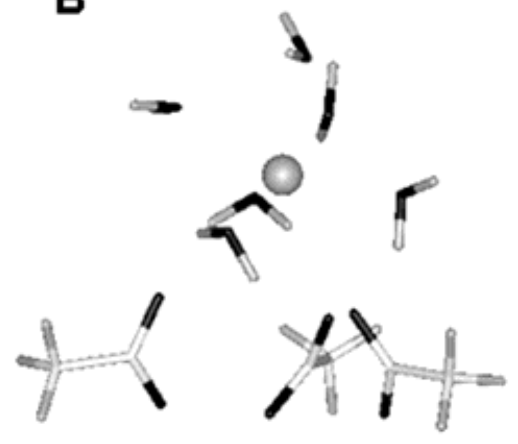

Fig. 2. Structures of the model calcium selectivity filter interacting with $\mathrm{Ca}^{2+}$ obtained from single ion calculations. Calcium ion (A) at $r=0 \AA$, bound form; (B) at $r=3.75 \AA$, the highest energy point and $(\mathbf{C})$ at $r=5.5$ $\AA$, the fully solvated ion (D). The structure of the model sodium selectivity filter with sodium ion bound at $r=0 \AA$.

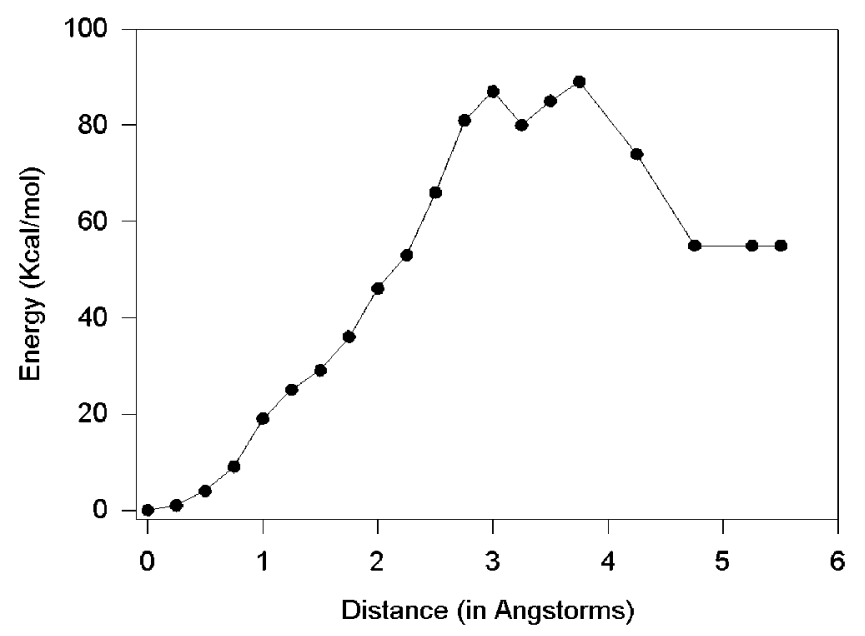

Fig. 3. Energy profile for single calcium ion interacting with MCSF.

fully bound to the flexible MCSF) to $3 \AA$, the energy increases smoothly. The energy remains near a maximum over a range of distances. Beyond $4 \AA$, the total energy decreases and attains a fairly constant value.

Analysis of the coordination shell of the cation in struc- tures optimized at various values of $\mathbf{r}$ reveals clear variations. As the ion moves away from the selectivity filter, the interaction between the cation and the flexible MCSF becomes less effective. The reduction in the energy is not adequately compensated by the additional interaction possible with the water molecules. Therefore, for small values of $\mathbf{r}$, the system is destabilized as $\mathbf{r}$ increases. At 3 $\AA$, only one oxygen per carboxylate ion interacts with the cation that is coordinated to four water molecules. Considerable reorganization of the coordination shell occurs for the structures with $\mathbf{r}$ in the range of $3-3.75 \AA$. Direct interaction with the flexible MCSF is essentially lost, and the water molecules in the first shell increase from four to six (Fig. 2B). The total energy remains roughly constant in this range of $\mathbf{r}$. However, at larger values of $\mathbf{r}$, the calcium ion is coordinated by seven water molecules (Fig. 2C).

The energetics associated with single ion permeation (Table I) can be extracted from the computed energy profile. The binding energy is explicitly defined as the difference between the initial state (fully solvated cation) and the final state (cation optimally bound to the selectivity filter). The large reduction in energy $(-55 \mathrm{kcal} / \mathrm{mol})$ as $\mathbf{r}$ goes from 5.5 to $0 \AA$ is a measure of the binding energy of the calcium ion and the flexible MCSF. Also, the ion has to 
TABLE I. Energetics of Single Ion Interactions With Various Model Selectivity Filters

\begin{tabular}{llcc}
\hline Model & $\begin{array}{c}\text { Activation } \\
\text { barrier for } \\
\text { desolvation } \\
\text { Cation } \\
\text { filter }\end{array}$ & $\begin{array}{c}\text { Binding energy } \\
(\mathrm{kcal} / \mathrm{mol})\end{array}$ \\
\hline $\mathrm{Ca}^{2+}$ & Flexible MCSF & 36 & -55 \\
$\mathrm{Na}^{+}$ & Flexible MSSF & 20 & -47 \\
$\mathrm{Na}^{+}$ & Rigid MCSF & 14 & -37 \\
$\mathrm{Na}^{+}$ & Flexible MCSF & 7 & -50 \\
\hline
\end{tabular}

overcome a barrier of $36 \mathrm{kcal} / \mathrm{mol}$ associated with the desolvation process.

A similar profile is obtained for the permeation of a single sodium ion across the flexible MCSF. The interaction energy between the cation and the core of carboxylate ions is large. In the bound form, the cation is coordinated to the anionic oxygen atoms and to an additional water molecule. At large values of $\mathbf{r}$, the cation is primarily coordinated to six water molecules. The process of binding to the selectivity filter involves an activation barrier associated primarily with the gradual restructuring of the coordinated water molecules around the cation. The precise magnitudes of the binding energy and the barrier depend on the geometric model chosen to describe the MCSF (Table II). With the rigid model calcium selectivity filter, in which the methyl groups of the acetate ions are fixed in the geometry of the MCSF bound to the calcium ion, the binding energy for the sodium ion is only -37 $\mathrm{kcal} / \mathrm{mol}$. When the constraints are removed allowing the acetate ions to optimize their interaction with the sodium ion, the value is, as expected, substantially higher. However, even using this flexible MCSF, the binding energy is calculated to be favorable for the calcium ion compared with the sodium ion by $-5 \mathrm{kcal} / \mathrm{mol}$. The barrier associated with the desolvation process is calculated to be lower for the sodium ion, both with the rigid and flexible MCSFs.

A highly stabilized structure is also obtained for the sodium ion interacting with the flexible model sodium selectivity filter. The binding energy relative to the structure in which the cation is entirely coordinated by water molecules is calculated to be $-47 \mathrm{kcal} / \mathrm{mol}$. The process of desolvation as the cation is brought toward the central core of the selectivity filter involves uneven energy changes. Interestingly, there is no significant overall activation barrier for binding, in contrast to the profiles obtained for single ion permeation in the MCSF.

A qualitatively different result is obtained for calcium ion interacting with the MSSF. Optimization from several reasonable initial geometries did not lead to a structure in which the cation is directly coordinated to the functional groups constituting the MSSF. Invariably, the cation becomes exclusively coordinated to the water molecules and is well separated from the selectivity filter.

The computed energy profiles for a single ion interacting with the model selectivity filters provide interesting insights (Table I). The MCSF is suggested to be capable of binding to both $\mathrm{Na}^{+}$and $\mathrm{Ca}^{2+}$. However, the binding energy is calculated to be more favorable for the calcium ion even with the flexible MCSF. The selectivity of the calcium channel can therefore be attributed to preferential binding of the native ion to the selectivity filter. A qualitatively different mechanism for ion selectivity is suggested for the sodium channel. The calcium ion does not bind to the model selectivity filter, whereas it has a sodium ion that has strong affinity for MSF. This implies that the selectivity in this case is through exclusion of ions that are not native to the channel.

The above interpretations are supported by the known transport characteristics of calcium and sodium channels. In the absence of calcium ions, calcium channels have been shown to conduct sodium ions. ${ }^{3}$ This has been interpreted by Kuo and $\mathrm{Hess}^{23}$ in terms of a conformational change at the selectivity filter. During sodium ion transport, the channel pore has been suggested to assume a nonselective conformation. The present study indicates that this is not essential. Even with the rigid MCSF, binding to the sodium ion is substantial. Interestingly, sodium channels are impermeable to calcium ions under all conditions. This is consistent with the exclusion model. Thus, the simple computational model correctly reproduces key differences in the mode of selectivity in these channels.

In quantitative terms, the model is inadequate. In particular, the binding energies and entrance barriers are large and are not compatible with the measured binding affinities or the flux rates. The choice of a small set of functional groups to represent a relatively complex channel pore, limitations in the extent of solvation that is explicitly included, errors associated with the force field that does not take into account polarization effects, inadequate search of the conformational space during geometry optimizations, and neglect of entropic contributions all could contribute to the discrepancy. These problems have been specifically recognized in previous modeling studies. $^{11}$

There is also a more fundamental difficulty in interpreting the results obtained for single ion transport. Although a large binding energy for the MSF for the native cation accounts for the selectivity, it also implies a large activation barrier for dislodging the ion from the bound state. Strong binding is clearly incompatible with large ion flux across the channel. Resolution of this paradox requires going beyond the assumption of the single ion mode of permeation through the selectivity filter. Because the ion concentration is fairly high in transport experiments, the permeation of an ion is likely to be influenced by the presence of another ion near the pore region. Therefore, we have explicitly evaluated the changes in the profiles associated with ion permeation when two ions interact with the model selectivity filters, as a simple model for multi-ion mode of ion transport.

\section{Energy Profiles for the Dual-Ion Mechanism}

The energetics associated with the permeation of a cation in the presence of a second ion was modeled for MCSF. For the case of a calcium ion dislodging another across the flexible MCSF (Table II), two parameters, $\mathbf{r}_{\mathbf{1}}$ 
TABLE II. Activation Barriers Along the Minimum Energy Pathway for Dual Ion Calculations in MCSF

\begin{tabular}{llc}
\hline & $\begin{array}{c}\text { Reaction coordinates at highest energy } \\
\text { structure }(\AA)\end{array}$ & $\begin{array}{c}\text { Activation energy } \\
(\mathrm{kcal} / \mathrm{mol})\end{array}$ \\
\hline Calcium replacing calcium & $r_{1}=2.0, r_{2}=-2.0$ & 21 \\
Sodium replacing sodium & $r_{1}=1.5, r_{2}=-3.0 ; r_{1}=3.0, r_{2}=-1.5$ & 17 \\
Sodium replacing calcium & $r_{1}($ sodium $)=3.0, r_{2}($ calcium $)=-4.5$ & 32 \\
Calcium replacing sodium & $r_{1}($ calcium $)=4.5, r_{2}($ sodium $)=-3.0$ & 29 \\
\hline
\end{tabular}

TABLE III. Reaction Coordinates and Relative Energies Along Minimum Energy Pathway for Sodium Displacing Sodium in Rigid MCSF

\begin{tabular}{ccc}
\hline $\begin{array}{c}\text { Sodium } \\
\left(r_{1} \text { in } \AA\right)\end{array}$ & $\begin{array}{c}\text { Sodium } \\
\left(r_{2} \text { in } \AA\right)\end{array}$ & $\begin{array}{c}\text { Relative energy } \\
(\mathrm{kcal} / \mathrm{mol})\end{array}$ \\
\hline 5.0 & -0.5 & 0 \\
3.0 & -1.5 & 17 \\
1.5 & -1.5 & 4 \\
1.5 & -3.0 & 17 \\
0.5 & -5.0 & 0 \\
\hline
\end{tabular}

TABLE IV. Reaction Coordinates and Relative Energies Along Minimum Energy Pathway for Calcium Displacing Calcium in MCSF

\begin{tabular}{ccc}
\hline $\begin{array}{c}\text { Calcium } \\
\left(r_{1} \text { in } \AA\right)\end{array}$ & $\begin{array}{c}\text { Calcium } \\
\left(r_{2} \text { in } \AA\right)\end{array}$ & $\begin{array}{c}\text { Relative energy } \\
(\mathrm{kcal} / \mathrm{mol})\end{array}$ \\
\hline 0.5 & -5.0 & 0 \\
1.0 & -3.5 & 19 \\
1.5 & -2.5 & 9 \\
2.0 & -2.0 & 21 \\
2.5 & -1.5 & 9 \\
3.5 & -1.0 & 19 \\
5.0 & -0.5 & 0 \\
\hline
\end{tabular}

and $\mathbf{r}_{2}$, corresponding to the distance of the ions to the core of the selectivity filter need to be specified. The first parameter was varied from 0 to $5 \AA$, where $0 \AA$ corresponds to the structure in which the cation is bound to the selectivity filter. The upper limit for $\mathbf{r}_{\mathbf{1}}$ was fixed at $5 \AA$, because the single ion profiles described above have shown that the cation is fully coordinated to water molecules beyond $4.75 \AA$. For each value of $\mathbf{r}_{\mathbf{1}}, \mathbf{r}_{\mathbf{2}}$ was varied from 0 to $\mathbf{r}_{\mathbf{1}}-5$. For every combination of $\mathbf{r}_{\mathbf{1}}$ and $\mathbf{r}_{\mathbf{2}}$, the positions of the carboxylate ions (except the methyl groups) and all the water molecules were optimized. These energies were used to generate a symmetric grid for all values $\mathbf{r}_{\mathbf{1}}$ and $\mathbf{r}_{\mathbf{2}}$, with $\mathbf{r}_{1}$ ranging from 0 to $5 \AA$, and $\mathbf{r}_{\mathbf{2}}$ ranging from -5 to $0 \AA$. The same strategy was used for computing the energetics for a sodium cation replacing another sodium ion bound to the MCSF (Table IV). The positions of the methyl groups in the acetate units were frozen in the geometry obtained by minimizing with a bound calcium ion. Hence, the model corresponds to sodium permeation through the rigid MCSF. Finally, the energetics was also calculated for the case in which the ions are dissimilar. The PE surface for a sodium ion displacing a calcium ion across the MCSF was obtained by varying $\mathbf{r}_{\mathbf{1}}$ from 0 to $5 \AA$ and $\mathbf{r}_{\mathbf{2}}$ from -5 to $0 \AA$ (Table V). To reemphasize, all the dual ion calculations including for
TABLE V. Reaction Coordinates and Relative Energies Along Minimum Energy Pathway for Sodium Displacing Calcium in Rigid MCSF

\begin{tabular}{ccc}
\hline $\begin{array}{c}\text { Sodium } \\
\left(r_{1} \text { in } \AA\right)\end{array}$ & $\begin{array}{c}\text { Calcium } \\
\left(r_{2} \text { in } \AA\right)\end{array}$ & $\begin{array}{c}\text { Relative energy } \\
(\mathrm{kcal} / \mathrm{mol})\end{array}$ \\
\hline 0.5 & -5.0 & 3 \\
3.0 & -4.5 & 32 \\
4.5 & -1.0 & 0 \\
\hline
\end{tabular}

assymetric ions, the initial geometry of MCSF was chosen after optimizing it for calcium binding (see section on computational methodology).

The energy surface for the multi-ion mechanism of calcium ion permeation through the flexible MCSF is shown in Figure 4A. The deep minima at the top right (facing the $\mathbf{r}_{\mathbf{2}}$ axis) near $(0,-5)$ and bottom left, near $(5,0)$ correspond to structures in which one cation is bound to the MCSF while the second is fully hydrated. The pathways along the axes, going from $(5,0)$ to $(5,-5)$ or $(0,-5)$ to $(5,-5)$, represent the single ion mechanism of permeation in the presence of a fairly distal second cation. These involve relatively high barriers, consistent with the results described in the previous section. Figure 4A clearly shows alternative favored pathways for replacement of one cation by another at the MCSF. The trajectory in which the bound cation moves away from the MCSF as the second ion approaches requires a substantially lower barrier (Tables II and III). In particular, the two equivalent minima near $(5,0)$ and $(0,-5)$ are smoothly connected via the intermediate structures with $\left(\mathbf{r}_{\mathbf{1}}, \mathbf{r}_{\mathbf{2}}\right)$ values of $(3,-1),(2.5,-1.5)$, $(2,-2),(1.5,-2.5)$ and $(1,-3)$. The overall activation barrier is estimated to be $21 \mathrm{kcal} / \mathrm{mol}$ along this path. At the highest energy structure, the two ions are relatively close to the MCSF at a distance of $2 \AA$. A range of other pathways are also conceivable across structures in which the cations are farther apart from each other. These involve barriers that are higher by $\geq 3 \mathrm{kcal} / \mathrm{mol}$.

The PE surface for the dual-ion mechanism for sodium ion transport across the rigid MCSF is shown in Figure 4B. As in the case of calcium ions, symmetrical minima are found at two extremes, with one ion being bound to the selectivity filter and the other fully hydrated. Although the preferred pathway for dislodging a sodium cation by another is also similar to that found for the dual-ion mode of calcium permeation, there are subtle differences. The intermediate structure in which both sodium cations interact with the rigid MCSF is a fairly deep minimum. The barrier for replacement of a sodium by another at the rigid 
MCSF is also lower for the sodium ions ( $\approx 17 \mathrm{kcal} / \mathrm{mol})$. Alternative paths noted for calcium ions involve significantly higher barriers for the case of sodium ions.

The computed energy grids for a calcium ion displacing a sodium cation from the MCSF and for the reverse process

A

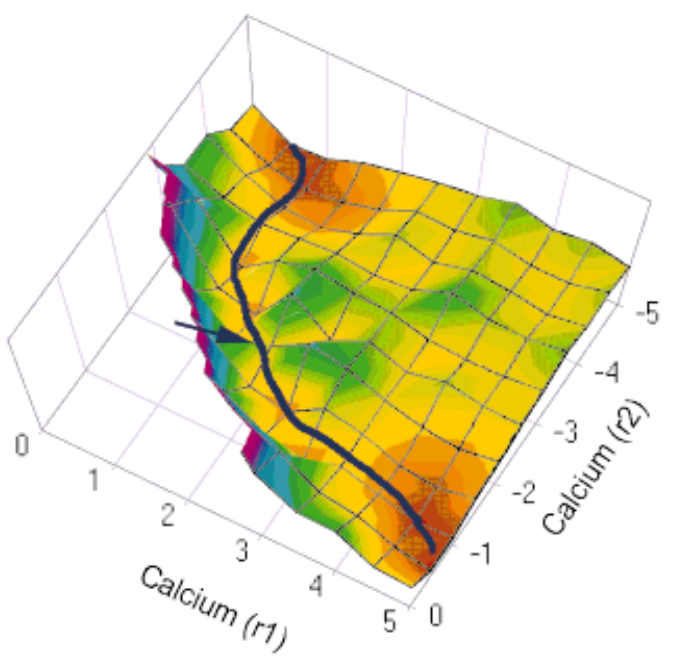

B

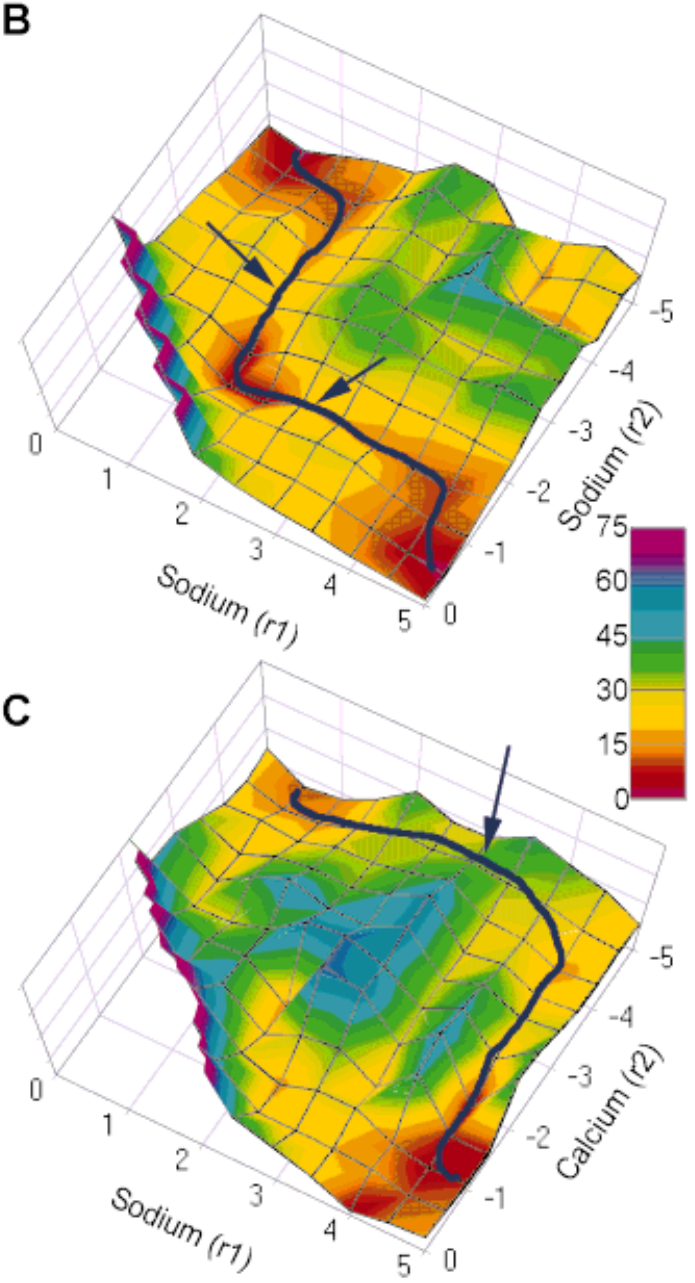

are quite revealing (Fig. 4B). The minimum, corresponding to the structure in which the calcium ion is bound to the selectivity filter and the sodium ion is hydrated, is more stable than the alternative minimum in which the sodium is coordinated to the MCSF and the calcium ion is exclusively solvated by water. The energy difference of 16 $\mathrm{kcal} / \mathrm{mol}$ is similar to the differential single ion binding energy of the two ions with the MCSF optimized for calcium binding. Two types of paths are available for interconversion between the two minima. The trajectory along which both ions interact strongly with the MCSF is less favored than the second pathway in which one of the ions drifts significantly away from the MCSF before the second ion moves in. The activation barrier along the most favorable pathway is $29 \mathrm{kcal} / \mathrm{mol}$ for the replacement of sodium by calcium and is $32 \mathrm{kcal} / \mathrm{mol}$ when calcium is replaced by sodium.

The calculated energetics for the dual-ion mode of ion transport through the MCSF has interesting implications. The replacement of a sodium cation by another at the MCSF requires the least barrier (Table II). In comparison, dislodgment of a calcium ion by another $\mathrm{Ca}^{2+}$ requires more energy. This is entirely consistent with the known behavior of calcium channels that not only permit sodium ion transport in the absence of calcium ions, but also at higher rates. ${ }^{3}$ When dissimilar metal cations are present, the calcium ion is predicted to displace a sodium ion more easily from the MCSF. The reverse process is calculated to involve a higher barrier. When this trend is combined with the calculated higher affinity of the MCSF for a calcium ion compared with a sodium ion, the exclusive transport of calcium ions through calcium channels under physiological conditions when both types of ions are found in abundance can be understood. The binding sites of channel pores are likely to be occupied preferentially by calcium ions (rather than by sodium ions) and consequently replaced more readily by calcium ions (again compared with sodium ions). The ability of calcium ions to block sodium ion transport through the MCSF is effectively accounted for by the present results. Thus, a unified interpretation of ion selectivity and flux rates is obtained.

Another notable feature of the activation barriers obtained for the dual-ion mechanism is that the values are substantially lower (range of 17-32 kcal/mol) than those computed by using the single ion permeation mechanism. The energy profiles obtained by using a single cation involve two high-energy steps. First, the hydration shell around the cation has to be restructured before optimum interaction with the selectivity filter becomes possible. In addition, the binding of the metal ion to the model

Fig. 4. Energy surface for dual-ion calculations. A: Calcium replacing calcium in MCSF. B: Sodium replacing sodium in rigid MCSF. C: Sodium replacing calcium in the rigid MCSF. The relative energies are appropriately color coded, and the scale bar indicating the relative energies is indicated in the figure. The regions of the plot with energies greater than $75 \mathrm{kcal} / \mathrm{mol}$ where the two ions are very close to each other have not been shown. A representative minimum energy path for ion displacement has been highlighted with a gray line. The arrows mark the position of activation barrier on the representative path. 
selectivity filter is calculated to be too strong. The combination of these two factors leads effectively to barriers in excess of $80 \mathrm{kcal} / \mathrm{mol}$ for the single ion mode of permeation. This brings us to one of the central paradoxes of ion transport through channels: large binding energy, which seems to be a requirement for high selectivity, is clearly incompatible with large flux rates. The dual-ion mechanism provides a partial resolution to this interpretational problem. Stabilizing interactions between the cation and the carboxylate ions emphasized by the single ion mode of conduction are evidently overwhelmed by intercation repulsion, which is taken into account in the dual-ion mechanism. Thus, the presence of a second ion effectively reduces the binding affinity, enabling efficient ion transport, but without compromising the selectivity for the native ion.

The present study provides some interesting insights concerning the dual-ion mode of ion transport. The precise details of how two-ion interactions are brought about within the channel pore have differed in the models postulated in recent years. Initially, it was assumed that more than one binding site, each hosting one ion, was involved, ${ }^{19}$, but mutagenesis experiments have detected only a single ring of residues constituting the selectivity filter. ${ }^{6}$ However, the likelihood of two ions simultaneously coordinated to the binding site at the selectivity filter has not been fully resolved. The present calculations confirm that it is indeed possible for two ions, especially a pair of sodium cations, and to a lesser extent a pair of calcium ions, to interact with the MCSF. Indeed, such structures are calculated to be involved in low-energy pathways available for ion conduction. Thus, the presence of a single binding site does not preclude the dual-ion mechanism of ion transport.

Although the calculated energetics provides a generally satisfactory interpretation of several observed trends in ion conduction, some problems still remain. The calculated barriers for ion transport are still fairly large, even with the dual-ion mechanism. Realistic estimates based on experimental conductances are only of the order of a few times $\mathrm{kT}$ and not in the range obtained in the present study. This is often a problem in modeling studies using empirical force fields, as noted earlier in investigations on gramicidin A. Although the nonpolarizable model may be partly responsible, the use of a single set of functional groups to model the channel pore is perhaps the principal reason for the high barriers obtained through calculations. A more detailed description of the selectivity filter with the full complement of functional groups may yield lower barriers. It is also likely that additional residues along the permeation path are needed to obtain more realistic barriers. Although not essential for binding and selectivity, the additional units may be postulated to principally help the desolvation process as the cation enters the channel pore. However, in the absence of precise structural details it would be difficult to quantify this hypothesis.

\section{CONCLUSIONS}

A simple computational model has been used to address two key questions concerning the permeation characteristics of calcium and sodium ion channels, i.e., the origin of selectivity for the native ion and the ability to conduct at high flux rates. A minimal set of functional groups was used to represent the MCSF and MSSF, based on mutagenesis experiments on these channels. Energy profiles obtained for a single ion moving from a fully hydrated structure to one in which it is essentially bound to the model selectivity filter suggest a clear distinction in the mode of selectivity in the two channels. Both calcium and sodium ions are calculated to have strong binding affinity for the MCSF, but the magnitude is larger for the calcium ion. In contrast, only the sodium cation is computed to bind effectively to the MSSF, resulting in a stable structure. These results imply that the selectivity in MCSF is primarily due to preferential binding for the calcium ion, whereas that of the MSSF is due to exclusion of the non-native cation from the selectivity filter. These conclusions are supported by experimental data that confirm that sodium transport is possible in calcium channels in the absence of calcium ions, whereas sodium channels are always impermeable to calcium ions.

Although a fundamental difference in the mode of selectivity in the two channels is captured by the energetics for single ion mode of transport, the computed barriers are inconsistent with the observed flux rates. Besides the high binding affinities, the process of partially desolvating the ion as it approaches the binding site of the selectivity filter introduces an additional barrier. As a way to overcome this interpretive problem, the possibility of two ions simultaneously interacting with the selectivity filter on opposite sides was therefore considered. The dual-ion mode of ion transport through the MCSF was indeed calculated to lead to substantially lower barriers. The relative activation energies are also generally consistent with the observed trends in permeation characteristics in calcium channels. The replacement of a sodium ion by another sodium is calculated to require the least energy, whereas dislodgment of a calcium ion by another calcium involves slightly higher energy. These are consistent with the higher flux rates for sodium transport (compared with calcium transport) through calcium channels, in the absence of calcium ions. Interestingly, the barrier for displacing a sodium ion from the bound state near the MCSF by a calcium ion is calculated to be easier than the reverse process.

The computed data can be combined to obtain a unified interpretation on ion transport characteristics of calcium channels. The selectivity filter has an intrinsic energetic preference to bind to calcium ions. Hence, at physiological concentrations, the MCSFs are occupied by calcium ions. The ions can be displaced relatively readily by other calcium ions, and not by sodium ions, resulting in efficient calcium transport.

Overall, the simple computational model captures many of the key features of calcium and sodium channels. However, the activation barriers for ion permeation are 
fairly large, even with the dual-ion mode of transport. This deficiency is presumably due to the highly simplified nature of the functional groups used to model the selectivity filters. In particular, inclusion of additional sites for coordination in the vicinity of the selectivity filter may contribute directly to the reduction of barriers associated with the desolvation process, but without having a bearing on the selectivity itself. ${ }^{24}$ It would be interesting to critically examine this idea in the potassium channel, for which precise structural information is available.

\section{ACKNOWLEDGMENTS}

The computations for the work were conducted at SERC, Indian Institute of Science. We would like to thank Mr. Sunthar at Chemical Engineering Department, IISc for helping us with the programs.

\section{REFERENCES}

1. Hille B. Ionic channels of excitable membranes. Sunderland, Massuchussets: Sinauer Associates Inc.; 1992

2. Bezanilla F, Armstrong CM. Negative conductance caused by entry of sodium and cesium ions into potassium channels of squid giant axons. J Gen Physiol 1972;60:588-608.

3. Almers WA, McCleskey EW. Non-selective conductance in calcium channels of frog muscle: Calcium selectivity in single file pore. J Physiol 1984;353:585-608.

4. Hess P, Tsien R. Mechanisim of ion permeation through calcium channels. Nature 1984;309:453-456.

5. Heinemann SH, Terlau H, Stuhmer W, Imoto K, Numa S. Calcium channel characteristics conferred on the sodium channel by single mutations. Nature 1992;356:441-443.

6. Yang J, Ellinor PT, Sather WA, Zhang J, Tsien, RW. Molecular determinants of calcium selectivity and ion permeation in L-type calcium channels. Nature 1993;366:158-161.

7. Roux B, Karplus M. Ion transport in a model gramicidin channel: structure and thermodynamics. Biophys J 1991a;59:961-981.

8. Roux B, Karplus M. Ion transport in a gramicidin-like channel: dynamics and mobility. J Phys Chem 1991b;95:4856-4868.

9. Roux B, Karplus M. Ion transport in gramicidin channel: free energy of the solvated right handed dimer in a model membrane. J Am Chem Soc 1993;115:3250-3260.

10. Roux B, Karplus M. Ion transport in gramicidin channel: molecu- lar dynamics study of single and double occupancy. Biophys $\mathrm{J}$ 1995;68:876-892.

11. Roux B, Karplus M. Molecular dynamics simulations of the gramicidin channel. Ann Rev Biophys Biomol Struct 1994;23:731761.

12. Favre I, Moczydlowski E, Schild L. On the structural basis for the ionic selectivity among $\mathrm{Na}^{+}, \mathrm{K}^{+}$, and $\mathrm{Ca}^{2+}$ in the voltage gated sodium channel. Biophys J 1996;71:3110-3125.

13. Ellinor PT, Yang J, Sater WA, Ahang, JF, Tsien RW. Calcium selectivity at a single locus for high affinity $\mathrm{Ca}^{2+}$ interactions. Neuron 1995;15:1121-1132.

14. Falke JJ, Snyder EE, Thatcher KC, Voertler CS. Quantitating and engineering the ion specificity of an EF-hand-like $\mathrm{Ca}^{2+}$ binding site. Biochem J 1991;30:8690-8697.

15. Doyle DA, Cabral JM, Pfuetzner RA, Kuo A, Gulbis JM, Cohen SL, Chait BT, MacKinnon R. The structure of the potassium channel: molecular basis of $\mathrm{K}^{+}$conduction and selectivity. Science 1998;280: $69-76$.

16. Kumpf RA, Dougherty DA. A mechanism for ion selectivity in potassium channel: computational studies of cation- $\pi$ interactions. Science 1993;261:1708-1710.

17. Lifson S, Hagler AT, Dauber P. Consistent force field studies of intermolecular forces in hydrogen bonded crystals. I. Carboxylic acids, amides and carbonyl H-bonds. J Am Chem Soc 1979;101: 5111-5121.

18. Hagler AT, Stern PS, Sharon R, Becker JM, Naider F. Computer simulation of the conformational properties of oligopeptides: comparison of theoretical methods and analysis of experimental results. J Am Chem Soc 1979;101:6842-6852.

19. Dauber-Osguthorpe P, Roberts VA, Osguthorpe DJ, Wolff J, Genest M, Hagler AT. Structure and energetics of ligand binding to proteins: Escherichia coli dihydrofolate reductase-trimethoprim, a drug-receptor system. Proteins 1988;4:31-47.

20. Beachy MD, Chasman D, Murphy RB, Halgren TA, Friesner RA. Accurate $a b$ initio quantum chemical determination of the relative energetics of peptide conformations and asssement of empirical force fields. J Am Chem Soc 1997;119:5908-5920.

21. Andersen OS, Koeppe RE. Molecular determinants of channel function. Physiol Rev 1992;72:S89-S158.

22. Chen XH, Bezprozvanny I, Tsien RW. Molecular basis of proton block of L-type $\mathrm{Ca}^{2+}$ channels. J Gen Physiol 1996;108:363-374.

23. Kuo CC, Hess P. Ion permeation through the L-type calcium channel in rat phaeochromocytoma cells: two sets of ion binding sites in the pore. J Physiol 1993;66:629-655.

24. Guidoni L, Torre V, Carloni P. Potassium and sodium binding to the outer mouth of the $\mathrm{K}^{+}$channel. Biochemistry 1999;38:85998604 . 